\title{
Adaptación cultural y validez del instrumento PIH para medir comportamientos de automanejo en cáncer
}

\author{
Cultural adaptation and validity of the instrument to measure self-management \\ behaviors in cancer
}

\section{Adaptação cultural e validade do instrumento pih para medir comportamentos de auto-gestão em câncer}

\author{
Gloria Mabel Carrillo González'; Oscar Javier Vergara Escobar² \\ 1 Doctora en Enfermería. Universidad Nacional de Colombia. Facultad de Enfermería. Sede Bogotá. gmcarrillog@unal.edu.co. orcid: \\ https://orcid.org/0000-0003-4513-104X \\ 2 Doctor en Enfermería, magíster en Enfermería. Universidad Nacional de Colombia. ojvergarae@unal.edu.co. orcid: https://orcid. \\ org/0000-0003-3158-9017
}

Recibido: 24/04/2021. Aprobado: 10/02/2021. Publicado: 5/04/2021

Carrillo-González GM, Vergara-Escobar OJ. Adaptación cultural y validez del instrumento pih para medir comportamientos de automanejo en cáncer. Rev. Fac. Nac. Salud Pública. 2021;39(2):e341816. Dor: https://doi.org/10.17533/udea.rfnsp.e341816

\section{Resumen}

Objetivo: Adaptar culturalmente al español y validar, en Colombia, el instrumento de comportamientos de automanejo para personas con cáncer. Método: Estudio transversal de validación de instrumento, en el que a partir de la versión original de la escala PIH [Patterns in Health scale, Patrones en salud], que mide los comportamientos de automanejo, se desarrolló su versión adaptada culturalmente al español y se adelantó la evaluación de la validez de constructo a partir de la aplicación de la escala a 182 adultos con diagnóstico de cáncer, para lo que se acudió a análisis factorial exploratorio y confiabilidad, mediante consistencia interna con el alfa de Cronbach. Resultados: Se obtiene un instrumento adaptado culturalmente al español en Colombia, para su uso en personas con cáncer. El análisis factorial arrojó cuatro factores con vector propio: conocimiento, reconocimiento y manejo de síntomas, alianza personal de salud/médico-paciente y afrontamiento. La consistencia interna es aceptable, con una alfa de Cronbach de 0,728. Conclusiones: La escala pih adaptada al español evalúa integralmente los comportamientos de automanejo de una persona con cáncer, y demostró validez de constructo y consistencia interna para su uso en el contexto colombiano en adultos con cáncer.

Palabras clave: Automanejo, enfermería oncológica, neoplasias, Patterns in Health (PIH) scale (escala Patrones en salud), psicometría. 


\begin{abstract}
Objective: Culturally adapt to Spanish and validate in Colombia the instrument of self-management behaviors for people with cancer. Method: Cross-sectional study of instrument validation in which, from the version of the PIH scale [patterns in health] that measures self-management behaviors, its culturally adapted version was developed in Spanish, construct validity was advanced from the application to 182 adults diagnosed with cancer. Exploratory factor analysis and reliability were used through internal consistency with Cronbach's alpha. Results: An instrument culturally adapted to Colombian Spanish is obtained for use in people with
\end{abstract}

cancer. The factor analysis revealed four factors with its own vector: knowledge, recognition and management of symptoms, alliance between health personnel / doctor-patient and coping. Internal consistency is acceptable with a Cronbach's alpha of 0.728. Conclusions: The PIH scale adapted to Spanish comprehensively evaluates the self-management behaviours of a person with cancer, demonstrated construct validity and internal consistency for use in the Colombian context.

-Keywords: Neoplasms, psychometrics, selfmanagement, oncology nursing, patterns in health (PIH) scale.

\section{Resumo}

Objetivo: Adaptar culturalmente o espanhol e validar na Colômbia o instrumento de comportamentos de autogestão para pessoas com câncer. Método: estudo transversal de validação de instrumento, no qual, a partir da versão da escala PIH [padrões em saúde] que mede comportamentos de autogestão, sua versão culturalmente adaptada foi desenvolvida em espanhol, a validade de construto foi avançada a partir do aplicação a 182 adultos diagnosticados com câncer. A análise fatorial exploratória e a confiabilidade foram utilizadas através da consistência interna com o alfa de Cronbach. Resultados: Um instrumento culturalmente adaptado ao espanhol colombiano é obtido para uso em pessoas com câncer. A análise fatorial revelou quatro fatores com seu próprio vetor: conhecimento, reconhecimento e manejo dos sintomas, aliança entre equipe de saúde / médico-paciente e enfrentamento. A consistência interna é aceitável com um alfa de Cronbach de 0,728. Conclusões: A escala PIH adaptada ao espanhol avalia de forma abrangente os comportamentos de autogestão de uma pessoa com câncer, demonstrou validade de construto e consistência interna para uso no contexto colombiano.

Palavras-chave: Neoplasias, psicometria, autogestao, enfermagem oncológica, escala padrões em saúde (PIH).

\section{Introducción}

El cáncer es una enfermedad que genera cambios en los estilos de vida, síntomas y efectos secundarios propios de las terapéuticas, algunos grados de dependencia y limitación, así como percepción de amenaza ante la muerte y temor. La experiencia de vivir en esta condición de cronicidad afecta tanto al enfermo como a la familia, con consecuencias en la calidad de vida, la adherencia, las complicaciones prevenibles e incluso altos costos para el sistema de salud [1].

Como otras enfermedades crónicas, el cáncer presenta características y dificultades asociadas al manejo de síntomas y discapacidad, al seguimiento de tratamientos complejos, el cambio de regímenes de alimentación y actividad física, la adaptación a las exigencias psicológicas y sociales, y el establecimiento de interacciones eficaces con los sistemas de atención en salud [2].

El modelo de cuidados crónicos es un referente que pone en la mesa de trabajo de políticas públicas, económicas y sociales, desde la Organización Mundial de la Salud y la Organización Panamericana de la Salud, la necesidad de generar modelos organizativos para el manejo de las enfermedades crónicas, dentro de estas el cáncer. Este modelo se centra en el automanejo de la condición de salud y en la relación de un paciente activado con un equipo de atención médica proactivo y preparado, lo que requiere de un sistema de salud apropiadamente organizado y vinculado con los recursos necesarios en la comunidad en general [3].

El automanejo se ha definido desde varias perspectivas. Sin embargo, Grady y Gough [4] lo conceptúan como la gestión diaria, por parte de los propios pacientes, de las enfermedades crónicas en el curso de estas. El automanejo se consolida como un área de interés mundial, porque representa una fusión de las metas del paciente, la familia, la comunidad y el equipo de salud, trabajando de manera conjunta para abordar mejor la enfermedad del individuo, al mismo tiempo que facilita el cuidado integral.

Ryan y Sawin [5] indican que es un proceso mediante el cual los individuos y las familias utilizan el conocimiento y las creencias, las habilidades de autorregulación y la facilitación social para lograr resultados 
relacionados con la salud, y pertenece a la dimensión del resultado proximal, y específicamente trata la condición, el riesgo o la transición de la salud. Adicionalmente, corresponde al manejo de síntomas, terapias farmacológicas y actividades respecto al tratamiento.

Dada la relevancia de la evaluación del automanejo, la Universidad de Flinders, en Australia [6], propone el desarrollo de un instrumento que mide el comportamiento de automanejo en enfermedades crónicas: el Partners in Health (PIH) scale, un programa que consiste en un conjunto genérico de herramientas y procesos de automanejo, basado en la terapia de comportamiento cognitivo, resolución de problemas y técnicas de entrevista motivacional personalizada.

La escala PIH [7] se desarrolló como instrumento genérico para usar junto con el paciente, frente a procesos de evaluación y establecimiento de objetivos. La versión inicial buscaba ser una herramienta corta y precisa, compuesta por 11 elementos autocalificados que reflejan la definición de autocontrol de la condición crónica. Posteriormente, esta definición incorporó un enfoque holístico del automanejo, con el objetivo de empoderar al individuo mediante estrategias proactivas en el manejo de los componentes físicos y psicosociales de su condición.

La escala original cuenta con propiedades psicométricas satisfactorias en cuanto a consistencia interna, confiabilidad entre evaluadores y una solución subyacente de tres factores de automanejo a partir del análisis factorial: automanejo central, conocimiento de la condición y monitorización de los síntomas. De esta forma, se expandieron los cinco principios de la condición crónica de automanejo a seis [7]: 1) conozca su condición y varias opciones de tratamiento; 2) negociar un plan de atención; 3) participar en actividades que protejan y promuevan su salud; 4) monitorear y manejar los síntomas y signos de la condición; 5) manejar el impacto de la condición en el funcionamiento físico, emociones y relaciones interpersonales, y 6) adoptar estilos de vida que promuevan la salud.

La validez de la versión original PIH de 12 ítems se confirmó en un estudio adelantado por Petkov et al. [8], en el que resultan cuatro factores: conocimiento de la enfermedad, afrontamiento con enfermedad, manejo de síntomas y adherencia a los tratamientos.

En el 2017, Smith et al. [9], con el propósito de evaluar la estructura factorial de la versión revisada, a partir de la aplicación de la escala a 904 personas con enfermedad crónica, plantean una modificación al instrumento en cuatro factores: conocimiento de enfermedad y tratamiento, alianza profesional paciente-salud, reconocimiento y manejo de síntomas, y afrontamiento.

En Latinoamérica, se resalta el trabajo adelantado por Peñarrieta et al. [10], quienes validan y adaptan culturalmente al español el instrumento PIH para el automanejo de enfermedades crónicas; la muestra estuvo conformada por 391 mujeres y 161 hombres, con edad promedio de 57 años. La distribución según diagnóstico médico fue hipertensión, diabetes y cáncer. Se adelantaron ajustes semánticos para mayor comprensión, sin cambiar el contexto original de las preguntas en inglés. Tres factores se derivaron del análisis exploratorio: la adherencia al tratamiento, el conocimiento de la enfermedad y el manejo de los signos y síntomas. Los elementos relacionados con adherencia y toma de decisiones compartida con los equipos de salud se fusionaron en el factor "adherencia".

Sin embargo, no se cuenta con una versión de la escala adaptada a Colombia, ni validada específicamente para adultos con cáncer que cursan con tratamientos oncológicos. Si bien el cáncer se considera una condición crónica, la modificación de estilos de vida, los síntomas que se presentan derivados de los tratamientos, así como la percepción y el estigma asociado a esta enfermedad ameritan un análisis particular respecto a otras condiciones crónicas. Tampoco se han analizado las diferencias en las dimensiones del instrumento frente a las validaciones adelantadas. Cabe señalar que se requiere contar con una escala que aporte como herramienta de automanejo del paciente con cáncer en tratamiento de quimioterapia o radioterapia, que permita orientar los planes de acción y de seguimiento frente a la condición de salud.

Con base en estos hallazgos, el objetivo del estudio fue adaptar culturalmente al español la escala PIH para adultos con cáncer, y validarla, en Colombia, en constructo y confiabilidad.

\section{Metodología}

Diseño descriptivo de corte transversal para validación de instrumentos, desarrollado entre los años 2018-2019. El estudio comprendió dos fases:

1. Adaptación cultural. Se hizo traducción directa al español, síntesis de traducción y retrotraducción del instrumento a su versión original, manteniendo la estructura. Estas traducciones estuvieron a cargo de dos traductores bilingües y del grupo investigador, a partir de la metodología para adaptación cultural propuesta por Beaton et al. [11]. Posteriormente, se adelantó una aplicación de la versión adaptada a 30 personas con cáncer, con diferentes características sociodemográficas y nivel educativo diverso, quienes evaluaron la comprensibilidad de la escala y realizaron observaciones a los ítems en cuanto a su semántica.

2. Validez de constructo y confiabilidad. Se aplicó la versión adaptada al contexto colombiano a una muestra de 182 pacientes con cáncer. Se llevó a cabo un análisis factorial exploratorio mediante análisis factorial de componentes principales, con el 
método de rotación Varimax, utilizando el paquete estadístico IBM ${ }^{\circledR}$ SPSS $^{\circledR}$ V26.0. (LICENCIA UNIVERSIDAD DE MCGILL).

Inicialmente, se examinó el índice de KaisserMeyer-Oilin de suficiencia, con el fin de estimar si un análisis de correspondencia era adecuado para los datos. Valores superiores a 0,7 en este índice se consideran aceptables para proceder con un análisis factorial. Posteriormente, de acuerdo con los resultados del análisis factorial de componentes principales, se estimaron índices de confiabilidad de alfa de Cronbach para cada una de las dimensiones observadas en la muestra.

No existe un acuerdo general sobre el tamaño de muestra requerido para un estudio de validación, aunque se recomienda un mínimo de 50 participantes [12].

\section{Población y muestra}

El estudio se adelantó en la Clínica Oncológica San Diego, en Bogotá, Colombia. Las personas que asisten a esta institución reciben algún tratamiento oncológico contra el cáncer. Se consideraron como criterios de inclusión: 1) mayores de 18 años, 2) con diagnóstico de cáncer, 3) en tratamiento oncológico con intención curativa y 4) con estado mental intacto. Se excluyeron pacientes en estado terminal o con deterioro cognitivo (determinado por el médico tratante).

Se reclutaron 182 pacientes, usando un muestreo a conveniencia. Los investigadores abordaron a los participantes en los servicios de consulta externa, a quienes se les explicó el objetivo del estudio y se adelantó el consentimiento informado. Posteriormente, se concertó una cita, en la que aquellos recibieron el cuestionario para su autodiligenciamiento en un espacio tranquilo dentro de la clínica. Los investigadores estuvieron atentos a apoyar a los pacientes en caso de dudas o inquietudes frente al formato.

\section{Consideraciones éticas}

El estudio contó con el aval del Comité de Ética Institucional de la Facultad de Enfermería de la Universidad Nacional de Colombia (Acta 13 del 12 septiembre 2017), así como del de los autores del instrumento en su versión original y en la versión adaptada al español, mediante correo electrónico.

Los pacientes recibieron explicación verbal del propósito del estudio y se obtuvo consentimiento informado para su participación.

Además, se acogieron los principios éticos establecidos en la Resolución 008430 de 1993, expedida por el Ministerio de Salud de Colombia [13].

\section{Resultados}

A continuación se describen los resultados derivados de cada una de las etapas.

\section{Adaptación cultural}

Se hizo traducción directa, síntesis de traducciones y retrotraducción. Se identificaron similitudes de todos los ítems en la traducción al español adelantada para Perú [10].

Acerca de la comprensibilidad del ítem, se realizaron modificaciones de contenido a 3 ítems, incluyendo la palabra "cáncer", para garantizar la adecuada interpretación del paciente frente a su condición oncológica.

Por otra parte, se ajustó el término "trabajador del área de la salud" por "personal de salud", por ser de mayor comprensión para la población colombiana.

Los ítems se redactaron con la afirmación en primera persona del singular, a fin de lograr, en la medida de las posibilidades, que sea el adulto quien diligencie y haga el autochequeo de sus comportamientos de automanejo. En la aplicación a 30 adultos con cáncer, la escala mostró alta comprensibilidad para los 12 ítems $(100 \%)$. La Tabla 1 muestra la versión original y la adaptada al español colombiano.

\section{Validez de constructo y confiabilidad}

Se describen las características de las personas que participaron en la validez de constructo y los hallazgos de esta fase.

\section{Caracterización de la muestra}

La muestra incluyó 182 personas (75,3\% mujeres) entre 19 y 75 años $\left(M_{\text {edad }}=51,08\right.$, desviación estándar $=12,35$ ), en su mayoría provenientes de los departamentos de Antioquia (29,7\%) y de Tolima (35,2\%).

Con respecto a la distribución por estrato socioeconómico (nivel económico; a mayor estrato, mejor el nivel económico y de acceso a los servicios públicos), se observó que el 59,90\% de los participantes pertenece a estratos 1 y 2 , seguido por el $39,60 \%$ en estratos 3 y 4 , y un $0,50 \%$ proveniente de estratos 5 y 6 .

Los diagnósticos oncológicos más frecuentes fueron el cáncer de mama (41,70\%), cáncer gástrico $(20,10 \%)$, cáncer de colon $(9,40 \%)$ y cáncer de ovario $(7,80 \%)$.

La mayoría de los participantes reportó bachillerato como el nivel educativo completado $(42,90 \%)$.

La Tabla 2 presenta otros estadísticos descriptivos de la muestra. 
Tabla 1. La Partners in Health (PIH) scale, versión adaptada para cáncer al español en Colombia

\begin{tabular}{|c|c|}
\hline Escala original & Escala adaptada para cáncer al español en Colombia \\
\hline 1. Overall, what I know about my health condition[s] is & 1. En general, lo que conozco acerca de mi estado de salud es \\
\hline $\begin{array}{l}\text { 2. Overall, what I know about my treatment, including } \\
\text { medications for my health condition[s] is }\end{array}$ & $\begin{array}{l}\text { 2. En general, lo que conozco acerca de mi tratamiento contra } \\
\text { el cáncer, incluyendo los medicamentos para el manejo de mi } \\
\text { condición de salud, es: }\end{array}$ \\
\hline $\begin{array}{l}\text { 3. I take medications or carry out the treatments asked by } \\
\text { my doctor or health worker: }\end{array}$ & $\begin{array}{l}\text { 3. Tomo los medicamentos y sigo las indicaciones dadas por el } \\
\text { médico o el personal de salud. }\end{array}$ \\
\hline $\begin{array}{l}\text { 4. I share in decisions made about my health condition[s] } \\
\text { with my doctor or health worker }\end{array}$ & $\begin{array}{l}\text { 4. Le comparto (informo) al médico o al personal de salud las } \\
\text { decisiones que tomo en cuanto a mi situación de salud. }\end{array}$ \\
\hline $\begin{array}{l}\text { 5. I am able to deal with health professionals to get the } \\
\text { services I need that fit with my culture, values and beliefs }\end{array}$ & $\begin{array}{l}\text { 5. Soy capaz de abordar a los profesionales de la salud para } \\
\text { obtener servicios que sean acordes con mi cultura, valores y } \\
\text { creencias. }\end{array}$ \\
\hline $\begin{array}{l}\text { 6. I attend appointments as asked by my doctor or health } \\
\text { worker }\end{array}$ & $\begin{array}{l}\text { 6. Asisto a las citas programadas por el médico o el personal } \\
\text { de salud que está a cargo de mi tratamiento contra el cáncer. }\end{array}$ \\
\hline $\begin{array}{l}\text { 7. I keep track of my symptoms and early warning signs } \\
\text { [e.g. blood sugar levels, peak flow, weight, shortness of } \\
\text { breath, pain, sleep problems, mood] }\end{array}$ & $\begin{array}{l}\text { 7. Me mantengo al tanto de los síntomas y signos de alerta } \\
\text { temprana (por ejemplo, los niveles de azúcar en sangre, límite } \\
\text { de presión arterial, peso, falta de aliento, dolor, problemas de } \\
\text { sueño, estado de ánimo). }\end{array}$ \\
\hline $\begin{array}{l}\text { 8. I take action when my early warning signs and symptoms } \\
\text { get worse }\end{array}$ & $\begin{array}{l}\text { 8. Tomo medidas ante las primeras señales de alerta y cuando } \\
\text { los síntomas empeoran. }\end{array}$ \\
\hline $\begin{array}{l}\text { 9. I manage the effect of my health condition[s] on my } \\
\text { physical activity [i.e. walking, household tasks] }\end{array}$ & $\begin{array}{l}\text { 9. Manejo los efectos que genera mi condición de salud en la } \\
\text { realización de actividades físicas (por ejemplo, caminar o hacer } \\
\text { tareas de la casa). }\end{array}$ \\
\hline $\begin{array}{l}\text { 10. I manage the effect of my health condition[s] on how I } \\
\text { feel [i.e. my emotions and spiritual well-being] }\end{array}$ & $\begin{array}{l}\text { 10. Manejo los efectos que genera mi condición de salud } \\
\text { sobre mi estado de ánimo (es decir, mis emociones y bienestar } \\
\text { espiritual). }\end{array}$ \\
\hline $\begin{array}{l}\text { 11. I manage the effect of my health condition[s] on my } \\
\text { social life [i.e. how I mix with other people] }\end{array}$ & $\begin{array}{l}\text { 11. Manejo los efectos que genera mi condición de salud sobre } \\
\text { mi vida social (es decir, la forma en que me relaciono con los } \\
\text { demás). }\end{array}$ \\
\hline $\begin{array}{l}\text { 12. Overall, I manage to live a healthy life [e.g. no smoking, } \\
\text { moderate alcohol, healthy food, regular physical activity, } \\
\text { manage stress] }\end{array}$ & $\begin{array}{l}\text { 12. En general, llevo un estilo de vida saludable (por ejemplo, } \\
\text { no fumar, consumo moderado de alcohol, alimentación sana, } \\
\text { actividad física regular, controlar el estrés). }\end{array}$ \\
\hline
\end{tabular}

Fuente: [9].

\section{Análisis factorial de componentes principales}

Después de examinar los estadísticos descriptivos de los ítems, estos se incluyeron en el análisis factorial exploratorio. El índice de Kaisser-Meyer-Oilin de suficiencia fue de 0,74 , indicando que el análisis de correspondencias era adecuado para los datos. De manera similar, el test Barlett de esfericidad arrojó un resultado estadísticamente significativo $\left(\chi^{2}[66]=511,69, p<0,000\right)$, lo que indica la existencia de correlaciones adecuadas entre los ítems, y que era pertinente proceder con el análisis.

El análisis arrojó como resultado cuatro factores con vector propio (eighenvalues) mayores que 1,00, y que en total lograron explicar el $61,14 \%$ de la varianza de las respuestas de los participantes.
El primer factor, denominado "conocimiento", está compuesto por dos ítems referentes al grado de entendimiento de los participantes acerca de su condición de salud y tratamiento. El segundo, denominado "reconocimiento y manejo de síntomas", incluyó tres ítems acerca del monitoreo de síntomas de alerta presente en la enfermedad y las medidas llevadas a cabo para controlarlos. El tercer factor, denominado "alianza personal de salud/ médico-paciente", abarcó dos ítems, que dan cuenta de la capacidad del paciente para comunicarse y trabajar en conjunto con los profesionales de la salud en pro de su mejoría. El cuarto factor observado, denominado "afrontamiento", cubrió cinco ítems, que describen las estrategias que el paciente implementa para hacer lleva- 
Tabla 2. Caracterización de los participantes en la validación de la escala de comportamientos de automanejo en cáncer

\begin{tabular}{|c|c|c|}
\hline \multicolumn{2}{|c|}{ Variable } & $\%[N=182]$ \\
\hline \multirow{2}{*}{ Género } & Masculino & 24,70 \\
\hline & Femenino & 75,30 \\
\hline \multirow{5}{*}{ Grado máximo de escolaridad } & Primaria & 26,40 \\
\hline & Bachillerato & 42,40 \\
\hline & Técnico / Tecnológico & 13,70 \\
\hline & Pregrado & 12,60 \\
\hline & Posgrado & 4,90 \\
\hline \multirow{3}{*}{ Estrato socioeconómico } & 1 y 2 & 59,90 \\
\hline & 3 y 4 & 39,60 \\
\hline & 5 y 6 & 0,50 \\
\hline \multirow{4}{*}{ Religión } & Católico & 80,20 \\
\hline & Cristiano & 14,80 \\
\hline & Testigo de Jehová & 0,50 \\
\hline & Ninguna & 4,50 \\
\hline \multirow{6}{*}{ Diagnóstico oncológico } & Cáncer de mama & 41,70 \\
\hline & Cáncer de colon & 9,40 \\
\hline & Cáncer de ovario & 7,80 \\
\hline & Cáncer gástrico & 20,10 \\
\hline & Cáncer testicular & 3,30 \\
\hline & Otros & 17,70 \\
\hline
\end{tabular}

dera su condición de salud en la vida diaria. En la Tabla 3 se presentan los resultados de dicho análisis.

A excepción de un ítem, en todas las preguntas del instrumento se observaron cargas factoriales superiores a 0,6 . El sexto ítem, "Asisto a las citas programadas por el médico o el personal de salud que está a cargo de mi tratamiento contra el cáncer", presentó cargas factoriales pobres, indicando que no corresponde a ninguno de los factores arrojados por el análisis. De acuerdo con ello, se llevó a cabo un segundo análisis factorial de componentes principales, en el cual no se incluyó esta pregunta, cuyo resultado presenta una estructura factorial compuesta por cuatro dimensiones: conocimiento, reconocimiento y manejo de síntomas, alianza médico-paciente y afrontamiento. En todos los ítems se observaron cargas factoriales adecuadas (superiores a 0,6 ). La estructura factorial logró explicar el $65 \%$ de la varianza en las respuestas de los participantes.
La estructura hipotética propuesta por Smith et al. [9] en 2017 coincide con los hallazgos de este estudio para pacientes con cáncer frente a los cuatro factores resultantes. Sin embargo, se observan discrepancias en algunos ítems que se ubican en los factores reconocimiento y manejo de síntomas (ítem 3), y en afrontamiento, el ítem 6 (véase Tabla 4).

\section{Análisis de confiabilidad}

Se estimó la consistencia interna de cada una de las dimensiones observadas, mediante alfa de Cronbach. Las dimensiones demostraron niveles aceptables de confiabilidad, así: para el instrumento $=0,728$; en conocimiento $=0,71$; reconocimiento y manejo de síntomas $=0,73$; alianza médico-paciente $=0,56$, y en afrontamiento $=0,69$. 
Tabla 3. Análisis factorial exploratorio de escala de automanejo (12 ítems). Matriz de componentes rotados (método Varimax).*

\begin{tabular}{ccccc}
\hline Ítem & \multicolumn{4}{c}{ Componente } \\
\hline & 1 & 2 & 3 & 4 \\
10 & 0,80 & 0,11 & 0,04 & 0,13 \\
11 & 0,74 & 0,10 & 0,05 & 0,03 \\
9 & 0,68 & 0,02 & 0,09 & $-0,03$ \\
12 & 0,55 & 0,33 & 0,03 & 0,22 \\
8 & 0,21 & 0,83 & 0,18 & 0,15 \\
7 & 0,20 & 0,77 & $-0,09$ & 0,13 \\
3 & $-0,01$ & 0,72 & 0,23 & 0,00 \\
1 & 0,23 & 0,17 & 0,82 & 0,07 \\
2 & 0,04 & 0,20 & 0,76 & 0,32 \\
4 & 0,13 & 0,12 & 0,07 & 0,80 \\
5 & 0,03 & 0,06 & 0,14 & 0,77 \\
6 & 0,37 & 0,16 & $-0,39$ & 0,29 \\
\hline * Se presentan resaltadas las cargas factoriales más altas de cada ítem.
\end{tabular}

Tabla 4. PIH-estructura propuesta por Smith versus versión PIH en cáncer adaptada culturalmente al español y validada para Colombia

\begin{tabular}{|c|c|c|}
\hline Factores de automanejo & $\begin{array}{l}\text { PIH [Estructura hipotética] para } \\
\text { enfermedad crónica propuesta } \\
\text { por Smith et al. (2017) }\end{array}$ & $\begin{array}{l}\text { Versión PIH en cáncer: adaptada al } \\
\text { español y validada para Colombia }\end{array}$ \\
\hline Conocimiento & Ítems 1 y 2 & Ítems 1 y 2 \\
\hline Alianza equipo salud-paciente en el tratamiento & Ítems 3, 4, 5 y 6 & Ítems 4 y 5 \\
\hline Reconocimiento y manejo de síntomas & Ítems 7 y 8 & Ítems 3, 7 y 8 \\
\hline Afrontamiento & Ítems $9,10,11,12$ & Ítems 9, 10, 11, 12 y 6 \\
\hline
\end{tabular}

\section{Discusión}

Este instrumento evalúa el automanejo de la enfermedad por una persona con cáncer, teniendo en cuenta su conocimiento sobre su condición, la negociación de planes de tratamiento con el equipo de salud, el manejo de síntomas y las implicaciones físicas, psicológicas y sociales que implican una condición crónica [7]. Se constituye en un aporte para abordar pacientes oncológicos en la población colombiana, con beneficios para estos, trascendencia para el personal de salud y de relevancia para la comunidad científica y la salud pública. Los resultados del estudio permiten afirmar que el instrumento es confiable y válido para ser aplicado en pacientes con cáncer.

Es de señalar que esta herramienta evalúa el automanejo en cualquier enfermedad crónica y puede ser autodiligenciada o aplicada por personal de salud. El automanejo se basa en el modelo conceptual de la Uni- versidad de Flinders [14] y se refiere a la capacidad de los pacientes para comprender la naturaleza de su condición y para gestionar y organizar el acceso a su cuidado. Los pacientes pueden reconocer señales tempranas de deterioro y tomar las medidas adecuadas, gestionar su estilo de vida para resultados óptimos de salud y trabajar de forma eficaz con los proveedores de atención médica [10].

El estudio presenta hallazgos similares en la adaptación cultural adelantada por Peñarrieta et al. [15] para el español, con modificaciones mínimas de contenido a 3 ítems, incluyendo la palabra "cáncer", para garantizar la adecuada interpretación del paciente frente a su condición de salud oncológica, ajuste al término "trabajador del área de la salud" por "personal de salud", y redacción con la afirmación en primera persona del singular.

Respecto a la estructura factorial resultante, el estudio coincide con los resultados reportados por Smith et 
al. [9], quienes en una población australiana y a través de análisis bayesianos revelan cuatro construcciones de afecciones crónicas, que mantienen una coherencia conceptual en línea con la práctica del automanejo de una condición crónica.

El factor 1 coincide con los ítems propuestos por Smith et al. [9] en la versión $2017 \mathrm{y}$ con lo descrito por Petkov et al. [8]. Este factor denota el conocimiento que tiene el individuo de su estado de salud y tratamiento. En tal sentido, un paciente que esté informado logrará un óptimo cuidado.

En este estudio, el factor 2, de alianza con el equipo de salud, coincide con la estructura propuesta por Smith et al. [9] en dos ítems. Estos hallazgos se asocian con la adherencia a las terapéuticas y a la prescripción médica [8].

Frente al factor 3, que corresponde al seguimiento de los signos y síntomas de la enfermedad, los ítems que se agrupan coinciden con la propuesta de Bodenheimer et al. [16]; sin embargo, se incluye uno más: "Tomo los medicamentos y sigo las indicaciones dadas por el médico o el personal de salud", resultados que evidencian que el paciente "pone en práctica" las orientaciones dadas por el personal de salud, siendo está una forma de manejar los síntomas que surgen en el cáncer.

El factor 4 es descrito como la capacidad de una persona para hacer frente al impacto físico y mental de su condición crónica. El estudio evidencia la estructura de este factor mediante los cuatros ítems que lo conforman, lo que se asocia con la comprensión de las perspectivas de afrontamiento, que incluyen un mundo externo/social de la persona, y uno interno, que corresponde a sus experiencias emocionales.

Ahora bien, este estudio es compatible con una de las tres subescalas que reporta Peñarrieta et al. [15] en la validación adelantada con una muestra de pacientes crónicos (diabetes, cáncer e hipertensión) peruana, la de conocimiento (ítems 1 y 2).

Lenferink et al. [17] encontraron una estructura de dos factores (conocimiento y afrontamiento, y adherencia al tratamiento) en una muestra de pacientes con enfermedad crónica obstructiva pulmonar en Holanda. Sus hallazgos no son compatibles con la estructura de cuatro subescalas de la versión original ni con la que resultó del presente estudio.

Smith et al. [18] utilizaron un agregado total de los ítems para representar un factor (involucramiento activo) en una muestra de pacientes en Australia, y encontraron una correlación positiva con una medida de percepción global de salud.

Es de señalar que se recomienda a los autores la revisión del ítem 6, el cual presentó cargas factoriales pobres. La eliminación de este ítem reportó, para este es- tudio, una estructura factorial compuesta por las cuatro dimensiones: conocimiento, reconocimiento y manejo de síntomas, alianza médico-paciente y afrontamiento, y logró explicar el $65 \%$ de la varianza en las respuestas de los participantes.

Las fortalezas del presente estudio están dadas por el abordaje hecho a pacientes con cáncer y el análisis de la validez estructural de la escala PIH revisada.

Dentro de las limitaciones es posible que existan diferencias respecto al tipo específico de tratamiento oncológico, en el componente de reconocimiento y manejo de síntomas.

Por otra parte, los pacientes pudieron haber tenido explicaciones dadas por el personal de salud institucional, que mejoraran sus conocimientos de automanejo, variable que no fue posible controlar.

Investigaciones futuras deben evaluar otras áreas de validez, como la de criterio, la capacidad de respuesta ante intervenciones propuestas, y la estabilidad frente a situaciones propias del tratamiento contra el cáncer y en los sobrevivientes.

\section{Conclusiones}

El instrumento PIH adaptado al español y validado en Colombia evalúa de forma integral los comportamientos de automanejo en personas con cáncer. Es una escala estructuralmente validada para medir construcciones relacionadas con el automanejo en pacientes con cáncer. Permite a los profesionales contar con una evaluación simple, que pueda conducir a planificar las intervenciones en personas que cursan con una condición oncológica.

\section{Agradecimientos}

A la Universidad Nacional de Colombia, Facultad de Enfermería, programa de Doctorado en Enfermería; a Colciencias, como centro y fuente de financiación, y en particular a los pacientes con cáncer que participaron en el estudio, ya que a través de su experiencia, cualificaron la investigación.

\section{Declaración de fuente de financiación}

Colciencias, Convocatoria 727. Doctorados Nacionales 2015. Universidad Nacional de Colombia, Dirección de Investigación Sede Bogotá.

\section{Declaración de conflictos de intereses}

Los autores declaran no tener conflictos de intereses. 


\section{Declaración de responsabilidad}

Los autores declaran responsabilidad de lo reportado en cada uno de los apartados del artículo

\section{Declaración de contribución por autores}

Los autores contribuyeron de forma sustancial en el diseño del artículo, participaron en el diseño del trabajo de investigación, intervinieron en la aprobación de la versión final que se publica y tienen la capacidad de responder todos los aspectos del artículo.

\section{Referencias}

1. Murillo R, Wiesner C, Acosta J, et al. Modelo de cuidado del paciente con cáncer. Instituto Nacional de Cancerología. Ministerio Salud y Protección Social [internet]; 2015 [citado 2017 mar. 15]. Disponible en: https://www.cancer.gov.co/files/libros/archivos/ modelo

2. Lorig K, Holman H. Self-management education: History, definition, outcomes, and mechanisms. Ann Behav Med. 2003;26(1):17. DoI: https://doi.org/10.1207/S15324796ABM2601_01

3. Organización Panamericana de la Salud, Organización Munida de la Salud. Cuidados innovadores para las condiciones crónicas: organización y prestación de atención de alta calidad a las enfermedades crónicas no transmisibles en las Américas [internet]; 2013 [citado 2017 mar. 18]. Disponible en: https://www.paho.org/ hq/dmdocuments/2013/CuidadosInnovadores-v5.pdf

4. Grady P, Gough L. El automanejo de las enfermedades crónicas: un método integral de atención. Rev. Panam Salud Publica [internet]. 2015 [citado 2017 mar. 18]; 37(3):187-94. Disponible en: https://scielosp.org/article/rpsp/2015.v37n3/187-194/

5. Ryan P, Sawin K. The individual and family self-management theory: Background and perspectives on context, process, and outcomes. Nurs Outlook. 2009;57(4):217-25. DOI: https://doi. org/10.1016/j.outlook.2008.10.004

6. Battersby M, Melanie $\mathrm{H}$, Lewis $\mathrm{R}$, et al. A randomised trial of the Flinders Program to improve patient self-management competencies in a range of chronic conditions: Study rationale and protocol. Australas. Medical J. 2010;1(3):198-204. Dor: https:// doi.org/10.4066/AMJ.2010.250

7. Battersby M, Ask A, Reece M, et al. The Partners in Health scale: The development and psychometric properties of a generic assessment scale for chronic condition self-management. Aust. J. Prim. Health. 2003;9(3):41-52. DoI: https://doi.org/10.1071/ PY03022

8. Petkov J, Harvey P, Battersby M. The internal consistency and construct validity of the partners in health scale: Validation of a patient rated chronic condition self-management measure. Qual
Life Res. 2010;19(7): 1079-85. DoI: https://doi.org/10.1007/ s11136-010-9661-1

9. Smith D, Harvey P, Lawn S, et al. Measuring chronic condition self-management in an Australian community: Factor structure of the revised Partners in Health [pih] scale. Qual Life Res. 2017;26(1): 149-59. DOI: https://doi.org/10.1007/s11136-0161368-5

10. Peñarrieta I, Barrios F, Gutierrez T, et al. Self-management inc chronic conditions: Partners in health scale instrument validation. Nurs Manag [Harrow]. 2014;20(10):32-7. Dor: https://doi. org/10.7748/nm2014.02.20.10.32.e1084

11. Beaton D, Bombardier C, et al. Guidelines for the process of cross-cultural adaptation of self-report measures. Spine. 2000;25(24):3186-91. DOI: https://doi.org/10.1097/00007632200012150-00014

12. Polit D, Yang F. Measurement and the measurement of change: A primer for the healh professions. Philadelphia: Wolters Kluwer Health; 2015.

13. Colombia, Ministerio de Salud. Resolución 008430 de 1993, por la cual se establecen las normas científicas, técnicas y administrativas para la investigación en salud (1993 oct. 4).

14. Lawn S, Battersby M, Harvey P, et al. A behavioural therapy approach to self-management: The Flinders Program. Diabetes Voice. 2009;54(Special Issue):30-32.

15. Peñarrieta $\mathrm{M}$, Vergel $\mathrm{O}$, Schimth $\mathrm{L}$, et al. Validación de un instrumento para evaluar el automanejo en enfermedades crónicas en el primer nivel de atención en salud. Rev. Cient. de Enferm. 2012;8(1):64-73.

16. Bodenheimer T, Lorig K, et al. Patient self-management of chronic disease in primary care. JAMA. 2002;288(19):2469-75. DOI: https://doi.org/10.1001/jama.288.19.2469

17. Lenferink A, Effing T, Harvey $P$, et al. Construct validity of the Dutch version of the 12-item partners in health scale: Measuring patient self-management behaviour and knowledge in patients with chronic obstructive pulmonary disease. PLoS one. 2016;11(8):116. DoI: https://doi.org/10.1371/journal.pone.0161595

18. Smith D, Lawn S, et al. Concurrent validity of the Partners in Health scale against general self-rated health in chronic conditions: A short report. Chronic IIIn. 2019;15(1):74-77. DOI: https:// doi.org/10.1177/1742395317743559 